\title{
Introduction. Émotion, empathie, affectivité. Les sujets et leur subjectivité à travers les pratiques langagières et éducatives
}

\author{
Luisa Fernanda Acosta Córdoba ${ }^{1, *}$ Vanessa Piccoli ${ }^{2, \dagger}$ \\ ${ }^{1}$ ICAR (UMR 5191), Université Lumière Lyon 2, 69007 Lyon, France \\ ${ }^{2}$ ICAR (UMR 5191), ENS de Lyon, 69007 Lyon, France
}

\section{Un sujet à multiples facettes}

Depuis 2015, le colloque pour jeunes chercheurs et chercheuses ICODOC réunit tous les deux ans de nombreux doctorants, doctorantes, jeunes docteurs et jeunes docteures pour discuter autour d'un thème transversal aux disciplines des sciences du langage et de l'éducation. Pour l'édition 2019, le comité d'organisation a proposé un espace de réflexion autour des émotions, thème pour lequel le laboratoire ICAR a été à l'avant-garde $[1 ; 2 ; 3$; $4 ; 5]$ et qui suscite un intérêt grandissant en sciences humaines.

Si aujourd'hui il peut paraître évident que dans des études qui s'intéressent aux interactions humaines, à la relation avec l'art ou aux apprentissages, la prise en compte de l'affectivité des sujets étudiés est primordiale, il y a seulement quelques années la place des émotions n'était souvent pas reconnue dans ces champs de recherche. Pour reprendre l'image très parlante évoquée par Swain, les émotions étaient : "the elephants in the room poorly studied, poorly understood, seen as inferior to rational thought" [6, p. 205]. C'est en effet seulement dans les années 2000 qu'en sciences humaines et sociales on assiste à ce qui a été nommé un affective turn [7], c'est-à-dire que les émotions ont été acceptées et revendiquées comme un objet d'étude à part entière.

Une fois ouverte la porte à l'étude de l'affectivité, ce champ de recherche s'est révélé être sans bornes. On ne se limite plus à repérer les émotions de base, telle que la tristesse, la colère ou la joie [8], mais on cherche à appréhender l'affectivité dans toutes ses nuances. On considère en effet que l'expression de l'émotion peut prendre de multiples formes et passer par de différents canaux. Elle peut bien évidemment être montrée, affichée par des mimiques et des postures, mais elle peut aussi être exprimée par le langage, par les textes, par les objets, de manière plus ou moins intentionnelle. Pour reprendre la tripartition de Micheli [9], l'émotion peut être dite, montrée ou étayée à travers une variété de procédés.

Dans toute étude qui s'intéresse à la communication, au langage, à l'intersubjectivité, la question de la place des émotions devient alors essentielle et peut se poser de manière transversale à tous les niveaux d'analyse. Comme Ochs et Schieffelin l'annonçaient dans leur étude pionnière : «Affect permeates the entire linguistic system. Almost any aspect of the linguistic system that is variable is a candidate for expressing affect » [10, p. 22].

\footnotetext{
*1uisa.acosta-cordoba@ens-lyon.fr

†vanessa.piccoli@ens-lyon.fr
} 
Si l'étude de l'affectivité peut être appliquée à tout domaine de recherche, certaines situations ont reçu une attention particulière en raison de la place spéciale qu'y occupent les émotions. C'est le cas notamment des contextes professionnels où un blocage émotionnel peut empêcher le bon déroulement de l'activité prévue. Ainsi, par exemple, de nombreux travaux se sont intéressés aux émotions des étudiants pour déterminer dans quelle mesure celles-ci entravent ou favorisent leur processus d'apprentissage, que ce soit en milieu scolaire [11] ou dans l'apprentissage d'une langue étrangère [12]. De manière semblable, un bon nombre d'études a été dédié à comprendre l'impact des manifestations d'empathie sur le déroulement des consultations médicales $[13,14]$. Par ailleurs, récemment, certains travaux ont montré les obstacles que la communication affective peut rencontrer dans des contextes plurilingues et interculturels, à cause d'une difficulté d'intercompréhension linguistique ou d'une conceptualisation des émotions différentes [15].

Par ailleurs, cet affective turn a mis en avant l'intérêt de prendre en compte non seulement les émotions des sujets étudiés, mais aussi l'affectivité de celles et ceux qui les étudient, c'est-à-dire des chercheurs et des chercheuses elles-mêmes. En effet, on a assisté à une forte prise de conscience de l'impact que la subjectivité de la personne a sur le processus de recherche lui-même. Comme le disent Schirrer et Schmitt : « si l'activité du chercheur semble en grande partie guidée par des considérations rationnelles, on ne peut passer sous silence qu'elle est aussi déterminée par la sensibilité du chercheur, son vécu et ses ressentis » [16, p. 249].

\section{Les contributions réunies dans ce volume}

La prise en compte des émotions se décline à travers plusieurs choix terminologiques, qui correspondent à des approches et traditions différentes. Ce volume témoigne de cette variété théorique et méthodologique, en proposant des contributions qui se situent en linguistique interactionnelle, analyse du discours, didactique des langues, sciences cognitives, sciences de l'éducation, sémiotique et typologie linguistique. Les quatorze contributions réunies dans ce volume ont été organisées en trois grands axes en fonction des terrains de recherche et des méthodologies employées par les auteurs.

\subsection{L'analyse des émotions en interaction}

Cette première partie contient quatre contributions où l'analyse des manifestations émotionnelles en interaction est mise en avant.

Le volume s'ouvre avec la contribution de Christian Plantin, chercheur émérite du laboratoire ICAR, un des conférenciers pléniers d'ICODOC 2019. Dans son article, Plantin résume certains des résultats de ses nombreuses études sur les émotions en interaction et leurs représentations. En prenant en compte la richesse lexicale du champ de l'affectivité, l'auteur propose plusieurs catégories analytiques permettant d'identifier les divers acteurs de l'émotion et la manière dont elle se propage au sein de l'interaction lors d'une séquence émotionnelle.

La deuxième contribution est une mise en application de l'étude des émotions à partir d'une analyse de cas tirée d'un corpus vidéo d'un débat informel en Colombie sur le féminisme. Luisa Acosta Córdoba mobilise les outils de l'analyse conversationnelle et multimodale pour observer l'émergence de l'identité femme-féministe en interaction, à partir de l'analyse séquentielle du rire.

Louis Maritaud présente également une analyse multimodale de corpus dans sa contribution. Dans cette étude sur le discours rapporté et l'expression de l'émotion vécue, l'auteur analyse la dimension syntaxique et gestuelle des réunions de relèves des équipes 
soignants en psychiatrie dans un hôpital français, afin d'observer comment l'expression des émotions prend place dans ce contexte médical.

La dernière contribution de cet axe porte sur l'expression des émotions dans un contexte professionnel : une émission radiophonique informative colombienne. Natalia Osorio Ruiz utilise plusieurs notions issues de l'analyse du discours et de l'analyse des interactions afin de contraster la communication émotive et la communication émotionnelle dans deux cas différents, qui illustrent les enjeux de cette situation communicative.

\subsection{Didactique, apprentissages, éducation}

La deuxième partie de ce volume réunit les cinq contributions qui ont pour sujet central l'apprentissage et les pratiques éducatives.

Dans la première contribution, Marie-Claire Lemarchand-Chauvin s'intéresse à la place des émotions chez les enseignants stagiaires d'anglais lors de leur année de formation initiale, en France. En adoptant un dispositif méthodologique en trois étapes (questionnaires, enregistrements vidéo de cours, entretiens d'auto-confrontation), l'autrice observe le rôle de la dimension émotionnelle dans cette première année d'expérience professionnelle en recensant les émotions éprouvées par les enseignants et en les mettant en lien avec leur production linguistique en cours.

La deuxième contribution porte sur les émotions chez les apprenants de l'anglais dans un lycée en France. Aurélie Bourdais aborde la question des enjeux affectifs qui accompagnent l'usage de traducteurs en ligne, en utilisant plusieurs méthodes comme l'enregistrement de l'écran pendant le processus de rédaction et les entretiens individuels. L'autrice explore ainsi les liens entre certaines émotions, comme l'embarras ou la frustration, et la motivation des lycéens.

Xin Zhang se penche également sur la dimension émotionnelle des apprenants, mais en contexte d'immersion. A partir d'un questionnaire réalisé auprès de 293 étudiants étrangers en France et grâce à une analyse statistique poussée, l'autrice pointe le rôle de l'apprentissage en milieu coopératif comme en facteur décisif dans le vécu émotionnel des apprenants de FLE.

La contribution de Sara Mazziotti porte quant à elle sur l'impact de la correction faite par les enseignants aux textes des élèves en CE2 et CM2, en France et en Italie. L'autrice analyse les indices d'affectivité de ces corrections et leur potentielle incidence dans le rapport que l'élève développe avec son texte, notamment dans sa décision d'abandonner sa production initiale.

La dernière contribution de cet axe s'intéresse aux événements émotionnels chez les jeunes enfants à la crèche et à l'école maternelle. Eva Feig propose un modèle, sous la forme d'un diagramme Kiviat, permettant d'évaluer différents facteurs qui interviennent dans l'événement émotionnel, comme l'intensité du ressenti enfantin et le traitement offert par l'adulte. Son modèle facilite la compréhension de situations communicatives complexes et plurilingues.

\subsection{Métaphores, discours, sémiotique}

Le dernier axe de ce volume rassemble cinq articles qui portent sur les différents modes de sémiotisation des émotions dans la langue et dans le discours.

Le premier travail propose une description approfondie des constructions lexicales qui expriment des émotions dans les langues hmong blanc et hmong noir. Léa Mouton présente une analyse sémantique et morphologique de ces psycho-collocations, qui, à partir de relations métaphoriques impliquant des parties du corps, servent à exprimer un grand nombre d'émotions dans ces langues d'Asie du Sud-Est. 
Imene Meriem Oumessad s'interroge sur la manière dont les émotions sont exprimées dans le discours journalistique français. L'autrice analyse un corpus d'articles du journal $L e$ Monde à propos de l'attentat contre Charlie Hebdo pour élucider les manières dont l'émotion y prend place, en distinguant entre l'émotion dite, l'émotion montée et l'émotion argumentée.

La contribution de Pauline Hachette s'intéresse aux émotions dans le cadre des études littéraires. En partant de la notion d'indisciplinarité, l'autrice problématise les divergences et convergences entre les courants formalistes et les courants herméneutiques. Dans cette réflexion sur les limites des sous-disciplines littéraires, l'autrice montre les enjeux de la lecture à partir de la prise en compte de la dimension affective.

Julien Thiburce présente une étude sur les émotions en mobilisant des notions issues de la sémiotique, de l'analyse conversationnelle et de l'analyse du discours. L'auteur propose une analyse de cas d'un corpus de balades urbaines guidées à Lyon, qui met en évidence non seulement la complexité de l'interaction analysée, mais plus largement, les enjeux épistémiques de l'étude des émotions.

La contribution qui clôt ce volume, proposée par une des conférenciers pléniers d'ICODOC 2019, porte sur une analyse sémiotique du rapport entre le langage visuel et les émotions. Maria Giulia Dondero, chercheuse au FNRS (Fonds National de la Recherche Scientifique), explore les liens entre la textualité, l'image, la gestualité et les émotions, en analysant les ouvrages de René Thom, Roland Barthes et Lev Manovich. L'autrice présente ainsi une réflexion approfondie sur le corps dans l'expérience émotionnelle liée à l'image.

\section{Bibliographie}

1. J. Cosnier. Psychologie des émotions et des sentiments (Retz, Paris, 1994).

2. C. Plantin. Les bonnes raisons des émotions (Peter Lang, Berne, 2011).

3. C. Plantin, M. Doury \& V. Traverso (Eds). Les Émotions dans les interactions (Presses Universitaires de Lyon, Lyon, 2000).

4. C. Polo, K. Lund, C. Plantin \& G. P. Niccolai. Group emotions: the social and cognitive functions of emotions in argumentation. International Journal of ComputerSupported Collaborative Learning, 11(2), 123-156 (2016).

5. M. Quignard, B. Ursi, N. Rossi-Gensane, V. André, H. Baldauf-Quilliatre, C. Etienne, C. Plantin \& V. Traverso. Une méthode instrumentée pour l'analyse multidimensionnelle des tonalités émotionnelles dans l'interaction. In F. Neveu et al. (Eds), 5e Congrès Mondial de Linguistique Française - CMLF 2016, SHS Web of Conferences 27, 15004 (2016).

6. M. Swain. The inseparability of cognition and emotion in second language learning. Language Teaching, 46, 195-207 (2013).

7. P. Clough \& J. Halley (eds). The Affective Turn: Theorizing the Social (Duke University Press, Durham, 2007).

8. P. Ekman, Are There Basic Emotions?, Psychological Review, 99, 550-553 (1992).

9. R. Micheli. Les émotions dans le discours. Modèle d'analyse, perspectives, empiriques. (De Boeck Supérieur, Louvain la Neuve, 2014).

10. E. Ochs \& B. Schieffelin. Language has a heart. The pragmatics of affect. Text, 9(1), 7-25 (1989).

11. A. Simöes-Perlant (ed). Numéro Spécial Emotions et Apprentissages Scolaires, ANAE 155, 30(4) (2018). 
12. I. Puozzo Capron \& E. Piccardo (eds). Numéro Spécial L'émotion et l'apprentissage des langues, Lidil 48 (2013).

13. J. Ruusuvuori. Managing al̈ect: integration of empathy and problem-solving in health care encounters. Discourse Studies 9(5), 597-622 (2007).

14. R. Wynn \& M. Wynn. Empathy as an interactionally achieved phenomenon in psychotherapy. Characteristics of some conversational resources. Journal of Pragmatics 38, 1385-1397 (2006).

15. M. T. Prior \& G. Kasper (eds). Emotion in Multilingual Interaction (John Benjamins Publishing Company, Amsterdam, 2016).

16. M. Schirrer \& D. Schmitt. Les émotions dans l'activité de recherche. Le cas de la conduite d'entretiens. Corps, 14(1), 249-258 (2016). 